\title{
Efectos de la competencia del terapeuta en el tratamiento de adolescentes con fobia social generalizada
}

\author{
Pablo J. Olivares-Olivares ${ }^{1 *}$, Luis Montesinos ${ }^{2}$, Ana I. Rosa-Alcázar ${ }^{1}$, Diego Macià ${ }^{3}$ y Maravillas Amorós ${ }^{1}$ \\ 1 Universidad de Murcia (España) \\ ${ }^{2}$ Montclair State University, New Jersey (USA) \\ ${ }^{3}$ Universidad Miguel Hernández de Elche (España)
}

\begin{abstract}
Resumen: El propósito de este estudio es verificar la parte de los resultados del Programa para la Intervención en Adolescentes con Fobia Social que puede ser atribuida a la competencia del terapeuta. Se presentan los resultados obtenidos en tres condiciones experimentales (grupos experto, cuasi-experto e inexperto), integradas por 46 adolescentes españoles con una edad media de 15.37 años $(D T=1.04$; rango $=14-18)$, siendo la mavoría chicas $(67.40 \%)$ que cursaban $4^{\circ}$ de E.S.O (46.70\%). Todos cumplieron los criterios requeridos para el diagnóstico de Fobia Social Generalizada. Los resultados muestran que (i) el tamaño del efecto que se puede atribuir a los terapeutas es bajo, (ii) que la magnitud del tamaño del efecto obtenido correlaciona con la magnitud de la competencia del terapeuta en las variables más sensibles al cambio terapéutico, (iii) que tales resultados no alcanzan diferencias con significación estadística entre las tres condiciones experimentales.

Los datos hallados nos permiten concluir que siendo relevante el impacto de la competencia del terapeuta en los resultados obtenidos la mayor parte de la varianza en los resultados se relaciona directamente con el efecto del programa de tratamiento manualizado que se ha aplicado.

Palabras clave. Grado de competencia del terapeuta; adolescentes; fobia social generalizada; programa IAFS.
\end{abstract}

Title: Therapist Effects in the Treatment of Adolescents with Generalized Social Phobia.

Abstract: The purpose of this study is to verify which part of the results of the Intervention Program for Adolescents with Social Phobia can be attributed to the level of competence of the therapist. We present the results of three experimental conditions (expert, quasi-expert and inexperienced), including 46 Spanish adolescents with a mean age of 15.37 years (SD= 1.04, range: 14-18). Most of the participants were female $(67.40 \%)$ and were in $4^{\text {th }}$ grade of ESO $(46.70 \%)$. All of them met the criteria for diagnosis of Generalized Social Phobia. The results showed that (i) the effect size that could be attributed to therapists was low, (ii) the magnitude of the effect size obtained correlated with the magnitude of the expert level in the most sensitive variables to therapeutic change, and (iii) these results did not reach statistically significant differences among the three experimental conditions.

The data allow us to conclude that found to be relevant the impact of therapist competence on the results most of the variance in the results is directly related to the effect of manualized treatment program that has been implemented.

Key words: Level of competence of therapist; adolescents; generalized social phobia; IAFS program.

\section{Introducción}

La Fobia Social se caracteriza por un miedo persistente y acusado a las situaciones sociales. Su tasa de prevalencia oscila entre el 3\% y el 13\% (APA, 2000), siendo el trastorno de ansiedad más frecuente tanto en población clínica como comunitaria, y ocupando el tercer lugar entre los de mayor prevalencia (Kessler et al., 2005).

Su tratamiento ocupa y preocupa a los investigadores tanto en población adulta como en la infancia y adolescencia (Olivares, 2009). Esta preocupación se fundamenta en su elevada prevalencia y las repercusiones que tiene para la salud física, la ocurrencia de otros trastornos comórbidos, el ajuste social o el rendimiento intelectual (Beidel y Turner, 2005; Olivares-Olivares, Rosa-Alcázar y Olivares, 2007).

Este interés ha producido tratamientos psicológicos que han mostrado su eficacia en el ámbito adulto e infanto-juvenil (véase Bados, 2009), hallándose entre los últimos la Intervención en Adolescentes con Fobia Social (IAFS; Olivares, 2005).

Los estudios realizados respecto del IAFS han probado su eficacia frente a grupos de control activos y pasivos y a otros tratamientos (Hernández, 2006; Olivares et al., 2003; Vallés, 2005), habiéndose iniciado también el estudio del pe-

* Dirección para correspondencia [Correspondence address]:

Pablo J. Olivares-Olivares. Departamento de Personalidad, Evaluación y Tratamiento Psicológicos. Facultad de Psicología, Campus de Espinardo, Universidad de Murcia. Apdo. 4021, 30100 Murcia (España). E-mail: pjoo1@um.es so de sus componentes (así Amorós, 2006 u Olivares, RosaAlcázar y Olivares-Olivares, 2006; Olivares-Olivares, RosaAlcázar y Olivares, 2008). Puede decirse que el IAFS es un tratamiento eficiente porque también ha mostrado mayores beneficios con menores costes que otros (Olivares et al., 2002) y ha sido replicado por un equipo independiente con buenos resultados (Gil-Bernal, 2009; Gil-Bernal y Hernández-Guzmán, 2009).

Por todo ello el presente estudio aborda la contribución del terapeuta en los resultados del IAFS, contextualizándose en un ámbito de investigación más amplio, el de las llamadas variables inespecíficas (Gavino, 2010) y, dentro de éstas, en el efecto que sobre los resultados del tratamiento puede tener el terapeuta (véase Kazantzis, 2003 o Wampold y y Bolt, 2006). Ahora bien, respecto del efecto o contribución del terapeuta en los resultados la información es muy contradictoria (véase Cella, Stahl, Reme y Chalder, 2011 u Okiishi et al., 2006). Así, mientras que Olivares et al. (2003) encontraron que los participantes tratados por psicólogos con mucha experiencia (dos o más años de ejercicio de la profesión en el tratamiento individual y grupal) producían los mejores resultados, dato coincidente con la posición de Wilkins, Nezu y Nezu (2001), otros estudios no confirman estos hallazgos (Kazantzis, 2003).

El objetivo del presente estudio es aportar evidencia sobre la medida en la que puede influir el nivel de competencia del terapeuta en los resultados generados por el Programa IAFS, de acuerdo con la información disponible al respecto (Kim, Wampold y Boltz, 2006) en el marco de una variabili- 
dad cuyo rango parece oscilar entre el $0 \%$ y el 50\% (CritsCristoph y Mintz, 1991).

\section{Método}

\section{Participantes}

La muestra la integraron 46 adolescentes. La edad media fue de 15.37 años (DT= 1.04; rango: $14-18)$, siendo la mayoría chicas $(67.4 \%)$ que cursaban $4^{\circ}$ de E.S.O (46.70\%). Se hallaron antecedentes familiares de Fobia Social para el 22\% y del $20 \%$ para otros problemas psicológicos. Ninguno había sido tratado previamente por problemas psicológicos. En la Tabla 1 se recogen otros datos relevantes.

\section{Procedimiento y diseño}

Se administró el SPAI y la SAS-A a 1.630 estudiantes de $3^{\circ}$ y $4^{\circ}$ de ESO y $1^{\circ}$ y $2^{\circ}$ de Bachillerato, en 6 institutos de la Región de Murcia, seleccionados aleatoriamente de un listado que incluía el total de centros públicos y concertados. Fueron excluidos $42(2.57 \%)$ por distintos motivos: haber recibido tratamiento psicológico previo, no cumplimentar o hacerlo inadecuadamente los cuestionarios administrados, haber cumplimentado solo uno de los dos cuestionarios, etc. La muestra definitiva fue de 1.588 participantes: 860 chicas (54.15\%) y 728 chicos (45.84\%). En la segunda fase, procedimos a la corrección de los cuestionarios y selección de los participantes a partir de los puntos de corte previamente determinados (SPAI $\geq 97$; SAS-A $\geq 57$; Olivares, GarcíaLópez, Turner, La Greca y Beidel, 2002); los superaron 124 $(11.7 \%)$. Éstos fueron evaluados mediante la entrevista ADIS-IV-C (Silverman y Albano, 1996; Silverman, Albano y Sandín, 2001); 52 cumplieron los criterios para el diagnóstico de Fobia Social Específica y 50 Fobia Social Generalizada. Se informó privadamente de los resultados de la evaluación. El requisito exigido fue presentar Fobia Social Generalizada y obtener el consentimiento escrito de uno o ambos padres; aceptaron $46(90 \%)$. En la tercera fase se realizó una sesión informativa con padres e hijos en la que se les dieron explicaciones y detalles pormenorizados respecto del Programa, aclarándoseles cualquier duda al respecto.

Tabla 1. Datos sociodemográficos.

\begin{tabular}{|c|c|c|c|c|}
\hline & & \multicolumn{3}{|c|}{ Grupo } \\
\hline & & $\begin{array}{c}\text { Experto } \\
(n=15)\end{array}$ & $\begin{array}{c}\text { Cuasiexperto } \\
(n=15)\end{array}$ & $\begin{array}{c}\text { Inexperto } \\
(n=16)\end{array}$ \\
\hline \multicolumn{2}{|l|}{ EDAD (media en años) } & 15.60 & 15.27 & 15.25 \\
\hline \multirow[t]{2}{*}{ GÉNERO $(\%)$} & HOMBRES & $4(26.7 \%)$ & $6(40.0 \%)$ & $5(31.3 \%)$ \\
\hline & MUJERES & $11(73.3 \%)$ & $9(60.0 \%)$ & $11(68.8 \%)$ \\
\hline \multirow[t]{3}{*}{ CURSO ESCOLAR } & $3^{\circ} \mathrm{ESO}$ & $33 \%$ & $47 \%$ & $38 \%$ \\
\hline & $4^{\circ} \mathrm{ESO}$ & $47 \%$ & $27 \%$ & $31 \%$ \\
\hline & $1^{\circ}$ Bachillerato & $20 \%$ & $26 \%$ & $31 \%$ \\
\hline \multicolumn{2}{|c|}{ NÚMERO DE SITUACIONES SOCIALES TEMIDAS/EVITADAS } & 6.73 & 5.73 & 6.31 \\
\hline \multirow{12}{*}{ COMORBILIDAD (\%) } & Trastorno de pánico & $4(26.70 \%)$ & $4(26.70 \%)$ & $3(18.80 \%)$ \\
\hline & Agorafobia & $1(6.70 \%)$ & $2(13.30 \%)$ & $1(6.30 \%)$ \\
\hline & Mutismo selectivo & $2(13.30 \%)$ & $2(13.30 \%)$ & $2(12.30 \%)$ \\
\hline & $T A G^{1}$ & $4(26.70 \%)$ & $4(26,70 \%)$ & $5(31.30 \%)$ \\
\hline & TOC ${ }^{2}$ & $0(0.00 \%)$ & $0(0.00 \%)$ & $0(0.00 \%)$ \\
\hline & Fobia especifica & $5(33.30 \%)$ & $4(26.70 \%)$ & $6(37.50 \%)$ \\
\hline & TEP 3 & $0(0.00 \%)$ & $0(0.00 \%)$ & $0(0.00 \%)$ \\
\hline & Episodio depresivo mayor & $1(6.70 \%)$ & $1(6.70 \%)$ & $0(0.00 \%)$ \\
\hline & Trastorno distimico & $0(0.00 \%)$ & $3(20.00 \%)$ & $3(18.80 \%)$ \\
\hline & Abuso de alcohol & $0(0.00 \%)$ & $0(0.00 \%)$ & $1(6.30 \%)$ \\
\hline & AOST $^{4}$ & $1(6.70 \%)$ & $1(6.70 \%)$ & $0(0.00 \%)$ \\
\hline & $T_{P E^{5}}$ & $5(33.33 \%)$ & $4(26.70 \%)$ & $5(31.30 \%)$ \\
\hline \multicolumn{2}{|c|}{ ABANDONOS DEL TRATAMIENTO (porcentaje) } & $0.00 \%$ & $0.00 \%$ & $0.00 \%$ \\
\hline
\end{tabular}

${ }^{1} \mathrm{TAG}=$ Trastorno de ansiedad generalizada ${ }^{2} \mathrm{TOC}=$ Trastorno obsesivo compulsivo ${ }^{3} \mathrm{TEP}=$ Trastorno por estrés postraumático ${ }^{4} \mathrm{AOS} \mathrm{T}=\mathrm{Abuso}$ de otras sustancias tóxicas $\mathrm{TPE}=$ Trastorno de personalidad por evitación.

Optamos por un diseño experimental de dos factores con medidas parcialmente repetidas. El factor inter-sujetos fue el nivel de competencia del terapeuta que se operacionalizó siguiendo a Nezu y Nezu (2005) en tres grados:

(1) Experto: Psicólogo con más de dos años de experiencia laboral tratando casos individuales y aplicando tratamiento psicológico a grupos.
(2) Cuasiexperto: Psicólogo con más de un año y menos de dos de experiencia laboral durante el que ha tratado sólo casos individuales.

(3) Inexperto: Psicólogo sin experiencia en la aplicación de tratamiento psicológico.

A cada condición se le asignó aleatoriamente un terapeuta y un coterapeuta, de distinto sexo y con el mismo grado experto; ambos papeles fueron sorteados en cada pareja. 
El factor de medidas repetidas integró los datos de los momentos en las que se evaluaron los participantes.

Los 46 participantes se asignaron al azar, en cada grupo, a dos unidades de tratamiento (rango: 7-8 participantes).

Las sesiones de tratamiento se desarrollaron en espacios habilitados ad hoc en los centros educativos, en horario asequible a los alumnos y por las mañanas. No se produjo mortalidad experimental. Asistieron a todas las sesiones 45 $(98 \%)$ participantes y 1 (2\%) faltó a una sesión.

La integridad del tratamiento se controló mediante el manual del Programa IAFS (Olivares, 2005). Todos los aspirantes del estudio a formar parte como terapeutas fueron evaluados del conocimiento que tenían sobre el Programa IAFS, que un mes antes les fue entregado para su estudio. La evaluación se llevó a cabo mediante dos entrevistas realizadas por dos entrevistadores independientes, siguiendo un listado de cuestiones previamente confeccionado por dos expertos en la aplicación de cada una de las sesiones del IAFS sobre la estructura, objetivos, contenidos, actividades y formas de afrontar las dificultades que pueden surgir más frecuentemente en la aplicación de cada una de sus unidades de un tratamiento (véase Olivares, 2005). Para ser seleccionados se les requirió que cada entrevistador les hubiera otorgado una puntuación mayor o igual a 9 sobre 10 en el conocimiento del Programa IAFS. La fidelidad en el cumplimiento del Programa IAFS fue cuantificada, previo consentimiento escrito, mediante grabación audiovisual de las 12 sesiones que lo integran, las cuales fueron visualizadas por dos observadores independientes con amplia experiencia en su aplicación. Los observadores registraron en cada sesión el número de intervenciones que no se ajustaban al papel que se les asigna en el Manual. La fidelidad o adherencia fue del $96 \%$ y la fiabilidad inter-observadores de 0.95 y 0.97 para los terapeutas y co-terapeutas de ambos grupos, calculadas a partir del algoritmo: número de acuerdos/ número de acuerdos + número de desacuerdos.

El grado experto fue el mismo en cada pareja.

\section{Instrumentos}

Todos los participantes completaron en el pretest, postest y seguimientos los siguientes instrumentos de evaluación:

"Inventario de Ansiedad y Fobia Social" (The Social Phobia and Anxiety Inventory -SPAI-; Turner, Beidel, Dancu y Stanley, 1989). Olivares, García-López, Hidalgo, Turner y Beidel (1999) estudiaron sus propiedades en población adolescente española, obteniendo elevados coeficientes de consistencia interna: 0.95, 0.83 y 0.95 para los valores Fobia Social, Agorafobia y Diferencia, respectivamente.

"Escala de Ansiedad Social para Adolescentes" (The Social Anxiety Scale for Adolescents -SAS-A-; La Greca y López, 1998) validada en población adolescente española por Olivares et el., (2005), en cuyo estudio presentó una consistencia interna elevada para la Puntuación total (0.91), para el Miedo a la evaluación negativa (0.94) y superior a 0.40 para la correla- ción item-test. Para el presente estudio se adaptaron dos versiones, una para padres y otra para profesores, para cuantificar la validez social de la intervención. Ambas conservaron la misma estructura, contenido y número de ítems de la SAS-A.

"Cuestionario de confianza para hablar en público" (CCHP Personal Report of Confidence as Speaker, PRCS-; Gilkinson, 1942). Sus propiedades psicométricas en población adolescente española han sido estudiadas por Méndez, Inglés e Hidalgo, (1999). La escala presenta una alta consistencia interna (0.87) y una alta validez concurrente (0.87).

Self-statements during public speaking / Autoverbalizaciones durante una situación de Hablar en Público" (SSPS/AHP; Hofmann y DiBartolo, 2000) evalúa las respuestas cognitivas ante la situación de hablar en público. En una investigación dirigida por los profesores J. Olivares y M. D. Hidalgo (Rivero, 2005), con población adolescente española, se hallaron buenas propiedades psicométricas. Presenta alta consistencia interna $(0.90)$.

Cuestionario de Evaluación de Dificultades Interpersonales en la Adolescencia (CEDIA; Inglés, Méndez e Hidalgo, 2001). Su consistencia interna es de 0.94 y la fiabilidad test-retest de 0.84 para la Puntuación Total.

"Escala de Inadaptación" (EI; Echeburúa y Corral, 1987). Muestra excelentes propiedades psicométricas en el contexto clínico (Echeburúa, Corral y Fernández-Montalvo, 2000) alcanzando su coeficiente $\alpha$ de consistencia interna el valor de 0.94 .

"Escala de Autoestima" (Society and Adolescent Self Image SASI-; Rosenberg, 1965). Las propiedades psicométricas de esta escala en población adolescente española fueron estudiadas por Atienza, Moreno y Balaguer (2000) con buenos resultados. El valor de su coeficiente $\alpha$ de consistencia es de 0.92 y la fiabilidad test-retest de 0.85 .

Anxiety Disorders Interview Schedule for DSM-IV. Child Version (ADIS-IV-C; Silverman y Albano, 1996; Silverman, Albano y Sandín, 2001). Esta entrevista ha mostrado excelente frabilidad test-retest (Kappa $=.63-.80)$ e interjueces (0.82$.95)$ en su aplicación a los trastornos de ansiedad, incluida la Fobia Social (Silverman, Saavedra, \& Pina, 2001).

La evaluación fue realizada por 4 colaboradores previamente entrenados, agrupados en parejas de distinto sexo. Las evaluaciones fueron enmascaradas respecto del nivel de competencia de los terapeutas para evitar efectos de confundido.

Además se realizó una prueba observacional que se aplicó en el pretest, postest y en una medida de seguimiento a los 12 meses. Consistió en tres entrevistas con cada uno de los participantes, quienes debían de iniciar y mantener una conversación con un desconocido durante tres minutos; los temas fueron sus expectativas respecto del tratamiento psicológico (pretest), la valoración personal del tratamiento aspectos positivos, negativos y mejorables- (postest), y una descripción/valoración de las dificultades a las que habían tenido que enfrentarse y el modo en como las habían resuelto (seguimiento). En esta tarea participaron dos colaborado- 
res de distinto sexo, entrenados para mantener una postura neutra y a los que se instruyó para que no interactuaran con participantes de mismo sexo ni iniciaran ni mantuvieran la conversación.

La actuación de cada participante fue filmada con vídeocámara, previo consentimiento escrito de éstos y sus padres, registrándose y analizándose la duración total del contacto ocular (máximo 180 segundos) y el número de pausas mayores de 2 segundos durante la interacción oral. Las grabaciones fueron visualizadas y codificadas por dos observadores independientes, previamente entrenados y distintos a los evaluadores. Las correlaciones inter-jueces fueron altas: "Contacto Ocular" $\left(r_{\mathrm{xy}}=0.90\right)$ y Pausas de más de dos segundos $\left(r_{\mathrm{xy}}=0.92\right)$

\section{Tratamiento}

El Programa IAFS consta de 12 sesiones de tratamiento en grupo, de 90 minutos/sesión y periodicidad semanal. Sus elementos básicos son: componente educativo, entrenamiento en habilidades sociales, exposición y reestructuración cognitiva. Además incluye tareas para casa y prevención de recaídas (véase Olivares, 2005).

\section{Análisis estadísticos}

Para comprobar si los grupos estaban igualados (pretest) en las variables sociodemográficas se realizaron los análisis de las variables cuantitativas (análisis de varianza de un factor-ANOVAs-) y categóricas (pruebas de Chi-cuadrado]. Para verificar si el grado experto afectaba a los efectos de la intervención se realizó el análisis estadístico de las variables dependientes y de la significación clínica.

Para el análisis de las variables dependientes se realizaron ANOVAs de dos factores de medidas parcialmente repeti- das, siendo el factor intergrupo el grado experto y el intragrupo los momentos temporales (pretest, postest y seguimientos).

Para estimar la proporción de la varianza explicada por cada fuente de variación se calculó el índice eta cuadrado $\left(\eta^{2}\right)$.

Para el análisis de la significación clínica (véase Ogles, Lunnen y Bonesteel, 2001) valoramos a los participantes respecto de la reducción/eliminación del número de situaciones sociales temidas/evitadas informadas por ellos en el pretest, mediante un inventario que contenía las 12 más frecuentes (ADIS-IV-C), atendiendo a los criterios: (i) Recuperación (remisión al 100\%); (ii) Mejora/reducción entre el $75 \%$ y el $99 \%$.

Para comprobar si se presentaban diferencias respecto del grado experto utilizamos la prueba Chi-Cuadro sobre cada tabla de contingencia.

Los análisis se realizaron con el paquete SPSS 14.0 (2007).

\section{Resultados}

El estudio de la equiparación de los grupos mostró ausencia de diferencias estadísticamente significativas entre las condiciones experimentales.

Los resultados de las medias $(M)$, desviaciones típicas (DT) y los ANOVAs unifactoriales que fueron estadísticamente significativos están recogidos en la Tabla 2.

Sólo se presentaron diferencias estadísticamente significativas en el postest en dos variables: SPAI-Fobia Social y SPAI-Diferencia, que se mantienen a los 6 y 12 meses. En la variable SAS-A/Profesores las diferencias aparecen en la medida de seguimiento a los 12 meses. El resto no alcanzaron diferencias significativas y la magnitud de $\eta^{2}$ resultó irrelevante.

Tabla 2. Anova de las medidas de la ansiedad y sus correlatos que resultaron estadísticamente significativas.

\begin{tabular}{|c|c|c|c|c|c|c|c|}
\hline & & & $\begin{array}{l}\text { Experto } \\
(n=15)\end{array}$ & $\begin{array}{c}\text { Cuasi-experto } \\
\quad(n=15)\end{array}$ & $\begin{array}{c}\text { Inexperto } \\
(n=16)\end{array}$ & $F$ & $\mathrm{\eta}^{2}$ \\
\hline \multirow{3}{*}{\multicolumn{2}{|c|}{ Fobia Social }} & Pretest & $118.00(12.79)$ & $117.93(14.09)$ & $118.25(10.61)$ & $F(2.43)=0.003 ; p=.98$ & 0.00 \\
\hline & & Postet & $59.00(17.59)$ & $65.87(18.74)$ & $80.06(18.07)$ & $F(2.43)=5.462 ; p=.008$ & 0.20 \\
\hline & & Segto 6 & $50.53(16.52)$ & $61.60(15.78)$ & $70.19(21.24)$ & $F(2.43)=4.579 ; p=.02$ & 0.18 \\
\hline 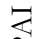 & & Segto 12 & $48.87(14.73)$ & $56.67(15.41)$ & $65.81(21.74)$ & $F(2.43)=3.567 ; p=.04$ & 0.14 \\
\hline \multirow[t]{4}{*}{$\frac{1}{\infty}$} & Diferencia & Pretest & $92.47(19.33)$ & $92.60(11.34)$ & $98.88(10.08)$ & $F(2.43)=1.055 ; p=.36$ & 0.05 \\
\hline & & Postet & $49.40(14.16)$ & $55.27(14.29)$ & $70.50(17.16)$ & $F(2.43)=7.900 ; p<.001$ & 0.27 \\
\hline & & Segto 6 & $42.00(16.76)$ & $54.00(14.58)$ & $62.50(20.78)$ & $F(2.43)=5.257 ; p=.009$ & 0.20 \\
\hline & & Segto 12 & $41.47(11.99$ & 49.87 (13.87) & $58.56(20.56)$ & $F(2.43)=4.406 ; p=.02$ & 0.17 \\
\hline \multirow{4}{*}{$\underset{\infty}{\stackrel{L}{L}}$} & Profesores & Pretest & $66.27(10.25)$ & $65.73(9.58)$ & $62.69(8.97)$ & $F(2.43)=0.633 ; p=.54$ & 0.03 \\
\hline & (Total) & Postet & $49.67(8.99)$ & $48.93(12.19)$ & $48.75(9.37)$ & $F(2.43)=0.034 ; p=.97$ & 0.002 \\
\hline & & Segto 6 & $37.00(6.43)$ & $38.20(7.83)$ & $43.00(10.25)$ & $F(2.43)=2.247 ; p=.12$ & 0.09 \\
\hline & & Segto 12 & $31.47(7.55)$ & $32.07(6.48)$ & $40.50(10.92)$ & $F(2.43)=5.405 ; p=.008$ & 0.20 \\
\hline
\end{tabular}

SPAI= Inventario de Ansiedad y Fobia Social SPAI-Fobia Social= Subescala de Fobia Social SPAI-Diferencia= Puntuación de diferencia

SAS-A= Escala de Ansiedad Social para SAS-A Profesores = Puntuación Total de la SAS-A/Versión para profesores

Los datos de las situaciones sociales temidas/evitadas, la duración total del Contacto ocular y el número de Pausas mayores de 2 segundos no alcanzaron diferencias con significación estadística. Sólo la "Duración del contacto ocular" las presentó en el postest y en la medida de seguimiento a los 12 meses (ver Tabla 3).

Para analizar el efecto del grado experto en su interacción con el momento temporal, realizamos ANOVAs de 
medidas parcialmente repetidas cuyos resultados presentamos en las Tablas 4, 5 y 6 .

Como podemos observar, las diferencias estadísticamente significativas se presentan en todas las medidas del Factor tiempo (programa de tratamiento); el grado experto [F (grupo)] no las alcanza en ninguna de las variables dependientes, a excepción del SPAI-Diferencia y marginalmente la Autoestima; la interacción sólo lo logra en las variables SPAI-Fobia Social, SAS-A Padres y SAS-A Profesores, pero con magnitudes de eta cuadrado muy próximas al límite de su valor significativo más bajo $\left(\eta^{2}<0.10\right)$.

Tabla 3. Anova de la Duración del contacto ocular.

\begin{tabular}{|c|c|c|c|c|c|c|}
\hline & & $\begin{array}{c}\text { Experto } \\
M(D T) \\
\end{array}$ & $\begin{array}{c}\text { Cuasi-Experto } \\
M(D T) \\
\end{array}$ & $\begin{array}{l}\text { Inexperto } \\
M(D T)\end{array}$ & $F$ & $\eta^{2}$ \\
\hline \multirow{3}{*}{ Duración del contacto ocular } & Pretest & $53.73(6.9)$ & $53.47(8.5)$ & $48.06(10.1)$ & $F(2,43)=2.143 ; p=.13$ & 0.09 \\
\hline & Postet & $103.93(31.8)$ & $88.46(27.3)$ & $69.50(20.6)$ & $F(2,43)=6.422 ; p=.004$ & 0.23 \\
\hline & Segto 12 & $104.86(33.4)$ & $91.86(30.0)$ & $77.31(27.4)$ & $F(2,43)=3.203 ; p=.05$ & 0.13 \\
\hline
\end{tabular}

M: Media, DT: Desviación Típica, $n$ : Tamaño del grupo $F=F$ de Snedecor $\eta^{2}=$ Eta cuadrado

Tabla 4. Anova mixto de las medidas de la ansiedad y sus correlatos.

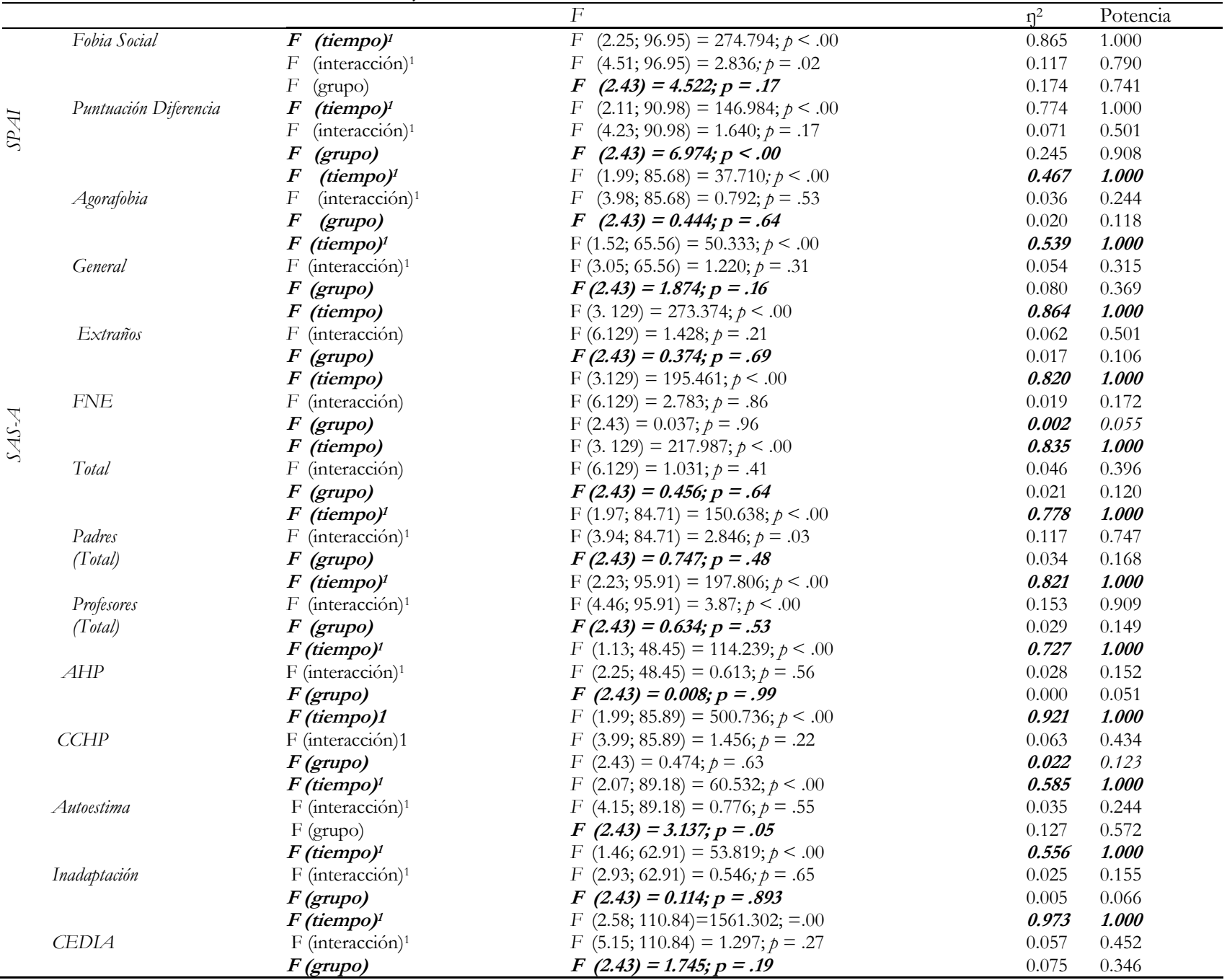

${ }^{1}$ Se aplicó la corrección de Greenhouse-Geisser al no cumplirse el supuesto de esfericidad.

SPAI= Inventario de Ansiedad y Fobia Social SPAI-Fobia Social= Subescala de Fobia Social SPAI-Diferencia= Puntuación de diferencia SPAIAgorafobia $=$ Subescala de Agorafobia general SAS-A = Escala de Ansiedad Social para Adolescentes SAS-A /SAD General $=$ Evitación y malestar ante la gente en general SAS-A/SAD Extraños= Miedo a la interacción con extraños SAS-A (FNE) = Subescala de Miedo a la Evaluación Negativa SAS-ATotal $=$ Puntuación total SAS-A- Padres $=$ Versión para padres SAS-A Profesores $=$ Versión para profesores AHP $=$ Cuestionario de Autoverbalizaciones al Hablar en Público CCHP = Cuestionario de Confianza para Hablar en Público CEDIA = Cuestionario para la evaluación de las dificultades interpersonales $\boldsymbol{M}=$ Media $\boldsymbol{D} \boldsymbol{T}=$ Desviación típica $\boldsymbol{F}=\mathrm{F}$ de Snedecor $\mathbf{y}^{2}=$ Eta cuadrado 
Tabla 5. Anova mixto de las medidas del número de situaciones sociales temidas/evitadas.

\begin{tabular}{|c|c|c|c|c|}
\hline & & $F$ & $\mathrm{y}^{2}$ & Potencia \\
\hline \multirow{9}{*}{ 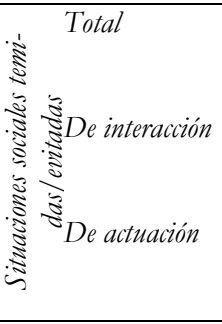 } & $F(\text { tiempo })^{1}$ & $F(1.55 ; 66.83)=213.444 ; p<.00$ & 0.832 & 1.000 \\
\hline & $F\left(\right.$ interacción) ${ }^{1}$ & $F(3.10 ; 66.83)=1.483 ; p=.23$ & 0.065 & 0.382 \\
\hline & $F$ (grupo) & $F(2.43)=1.132 ; p=.33$ & 0.050 & 0.236 \\
\hline & $F(\text { tiempo })^{1}$ & $F(1.39 ; 60.18)=174.662 ; p<.00$ & 0.802 & 1.000 \\
\hline & $F{\text { (interacción })^{1}}^{1}$ & $F(2.79 ; 60.18)=2.639 ; p=.06$ & 0.109 & 0.597 \\
\hline & $F$ (grupo) & $F(2.43)=0.426 ; p=.66$ & 0.019 & 0.115 \\
\hline & $F(\text { tiempo })^{1}$ & $F(1.54 ; 66.31)=74.263 ; p<.00$ & 0.633 & 1.000 \\
\hline & $F$ (interacción) $^{1}$ & $F(3.08 ; 66.31)=1.761 ; p=.16$ & 0.076 & 0.446 \\
\hline & $F$ (grupo) & $F(2.43)=1.864 ; p=.17$ & 0.080 & 0.367 \\
\hline
\end{tabular}

${ }^{1}$ Se aplicó la corrección de Greenhouse-Geisser al no cumplirse el supuesto de esfericidad. $\boldsymbol{F}=\mathrm{F}$ de Snedecor, $\mathbf{\eta}^{2}=$ Eta cuadrado

En la Tabla 5 podemos observar los resultados del ANOVA sobre las situaciones sociales temidas/evitadas. Estos muestran que ya se trate de situaciones sociales de interacción, de relación, o del conjunto de ellas, solo la variable Programa $[F($ tiempo)] alcanza nítidamente diferencias estadísticamente significativas en los tres casos, con magnitudes de eta cuadrado que van desde el $63.3 \%$ de la varianza hasta el $83.2 \%$.
El ANOVA para la "Duración total del contacto ocular" presentó un resultado estadísticamente significativo en el factor grupo, incrementándose los resultados favorables conforme mayor era el grado experto de los terapeutas. Sin embargo no se encontraron diferencias entre los grupos cuando se analizó el número de pausas mayores de 2 segundos (véase Tabla 6). El Programa de tratamiento es la única variable que alcanza significación estadística siendo las magnitudes de eta cuadrado altas.

Tabla 6. Anova mixto de las medidas del contacto ocular y del número de pausas mayores de dos segundos.

\begin{tabular}{|c|c|c|c|c|}
\hline & & $F$ & $\eta^{2}$ & Potencia \\
\hline \multirow[t]{3}{*}{ Duración del contacto ocular } & $F(\text { tiempo })^{1}$ & $F(1.47 ; 63.25)=66.551 ; p<.00$ & 0.865 & 1.000 \\
\hline & $F$ (interacción) $^{1}$ & $F(2.94 ; 63.25)=2.673 ; p=.06$ & 0.111 & 0.620 \\
\hline & $F$ (grupo) & $F(2.43)=5.644 ; p<.01$ & 0.208 & 0.836 \\
\hline \multirow[t]{3}{*}{ Número de pausas mayores de dos segundos } & $F(\text { tiempo })^{1}$ & $F(1.56 ; 67.36)=138.923 ; p<.00$ & 0.764 & 1.000 \\
\hline & $F\left(\right.$ interacción) ${ }^{1}$ & $F(3.13 ; 67.36)=0.730 ; p=.54$ & 0.033 & 0.201 \\
\hline & $F$ (grupo) & $F(2.43)=0.686 ; p=.51$ & 0.031 & 0.158 \\
\hline
\end{tabular}

${ }^{1}$ Se aplicó la corrección de Greenhouse-Geisser al no cumplirse el supuesto de esfericidad.

$\mathbf{F}=\mathrm{F}$ de Snedecor. $\boldsymbol{\eta}^{2}=$ Eta cuadrado

Los datos recogidos en la Tabla 6 muestran que el efecto del Programa a lo largo del tiempo resultó significativo en ambas variables, indicando una importante mejoría del pretest al postest y algo más baja del postest al seguimiento a los 12 meses. En cambio, la interacción no resultó estadísticamente significativa, aun cuando el resultado de la "Duración total del contacto ocular" fue marginalmente significativo ( $p$ $=.056$ ) pero su eta cuadrado representa el $11.1 \%$ de la varianza explicada.

La significación clínica fue determinada mediante el número de sujetos que presentaban una disminución de las situaciones sociales temidas/evitadas e informadas en el pretest. Distinguimos dos categorías: Recuperación (dejar de cumplir los criterios para el diagnóstico de Fobia Social) y Mejoría (reducción entre el 75\% y el 99\%). El estudio de recuperación muestra ausencia de diferencias estadísticamente significativas entre los grupos en el postest y en los seguimientos a 6 y 12 meses. No obstante, se observa que en el grupo tratado por terapeutas expertos los participantes se recuperan más que en los otros grupos (véase la Tabla 7).

Los datos de mejoría al 75\%-99\% tampoco muestran diferencias significativas entre los grupos, ni en el postest ni en los seguimientos a los 6 y 12 meses, pese a que se observe un incremento sostenido de éstas en los grupos Inexperto y Experto entre el postest y el primer seguimiento.

Tabla 7. Resultados de eficacia del tratamiento en tantos por ciento.

\begin{tabular}{lcc}
\multicolumn{2}{l}{ Tabla 7. Resultados de eficacia del tratamiento en tantos por ciento. } \\
\hline $\begin{array}{l}\text { Remisión } \\
100 \%(n)\end{array}$ & $\begin{array}{c}\text { Mejoría } \\
75 \%-99.99 \%(n)\end{array}$ \\
Medidas & $53.33 \%(8)$ & $13.33 \%(2)$ \\
\hline Postratamiento & $60.00 \%(9)$ & $20.00 \%(3)$ \\
$\quad$ Experto & $37.50 \%(6)$ & $18.75 \%(3)$ \\
$\quad$ Cuasiexperto & & \\
$\quad$ Inexperto & $73.30 \%(11)$ & $0.00 \%(0)$ \\
Seguimiento a los 6 meses & $60.00 \%(9)$ & $6.66 \%(1)$ \\
$\quad$ Experto & $43.75 \%(7)$ & $12.50 \%(2)$ \\
$\quad$ Cuasiexperto & & \\
$\quad$ Inexperto & $73.30 \%(11)$ & $13.33 \%(2)$ \\
Seguimiento a los 12 meses & $60.00 \%(9)$ & $26.66 \%(4)$ \\
$\quad$ Experto & $50.00 \%(8)$ & $12.50 \%(2)$ \\
$\quad$ Cuasiexperto & & \\
Inexperto &
\end{tabular}

\section{Discusión}

Nuestros resultados muestran que, con excepción de algunas variables muy sensibles al cambio terapéutico, como el "Contacto Ocular", el perfil estadístico de los datos obtenidos por los tres grupos al aplicar el Programa es muy parecido, es decir, los efectos producidos por la aplicación del 
Programa no difieren significativamente con independencia del quién lo aplique; las bajas proporciones de varianza explicada por el nivel o grado de competencia del terapeuta también lo corroboran (véanse tablas 4, 5 y 6). De hecho la relevancia del efecto del Programa es la que permite explicar el porqué a los 12 meses de terminado el tratamiento, incluso los terapeutas inexpertos logran que la mitad de los miembros de su grupo de tratamiento se recuperen. Datos que concuerdan con los hallazgos de Elkin, Falconnier, Martinovitch y Mahoney (2006), y nos permite indicar que, en nuestro estudio, el efecto atribuible a la competencia del terapeuta en los resultados se sitúa en la parte baja del rango informado por Crits-Cristoph y Mintz (1991).

Este hallazgo también se constata en los datos proporcionados por una de las medidas que se muestran más sensibles al cambio terapéutico, el Contacto ocular.

No obstante, aunque la parte de la varianza de los resultados que se puede atribuir a los terapeutas es pequeña, comparada con la del efecto del Programa, en línea con lo informado, por ejemplo, por Wiborg, Knoop, Wensing, \& Bleijenberg (2013), dentro de ésta el efecto del terapeuta también se relaciona con su grado o nivel de competencia. Así, el estudio de los promedios obtenidos, por ejemplo, en la Subescala de Fobia Social del SPAI y en su Puntuación Diferencia, muestra que los participantes tratados por psicólogos expertos mejoran generalmente más que los de cuasiexpertos y éstos que los de los inexpertos (véanse las tablas 2 y 3). Otro tanto ocurre en los resultados relativos a la remisión de la FS a tendiendo a criterios clínicos (véase Tabla 7), es decir, al número de participantes que dejan de cumplir los criterios diagnósticos. En este caso los expertos y los cuasiexpertos obtienen mejores resultados que los inexpertos en el postest, y en las medidas de seguimiento a los 6 y 12 meses. Estas diferencias se podrían explicar atendiendo a los resultados del estudio realizado por Anderson, Ogles, Patterson, Lambert, y Vermeersch (2009), en el que se pone de manifiesto tanto la relación entre la experiencia del terapeuta y las habilidades interpersonales como la capacidad predictora de estas últimas respecto de la eficacia del terapeuta en la aplicación de los tratamientos psicológicos, debido a su papel a la hora de afrontar y resolver exitosamente problemas interpersonales durante la aplicación del tratamiento. De hecho el grado de competencia de los terapeutas se asocia con mayor eficacia a la hora de tratar a los pacientes más graves (Strunk, Brotman, DeRubeis, y Hollon, 2010).

\section{Referencias}

American Psychiatric Association. (2000). Diagnostic and Statistical Manual of Mental Disorders (cuarta edición, texto revisado). Washington, DC: APA.

Amorós, M. (2006). Efectos de la eliminación de los componentes de reestructuración cognitiva y focalización de la atención en la eficacia de la Intervención en Adolescentes con Fobia Social. Tesis doctoral no publicada. Facultad de Psicología. Universidad de Murcia.

Anderson, T., Ogles, B. M., Patterson, C. L., Lambert, M. J., y Vermeersch, D. A. (2009). Therapist effects: Facilitative interpersonal skills as a predictor of therapist success. Journal of Clinical Psychology, 65 (7), 755-768.

\section{Conclusiones y limitaciones}

A la luz de nuestros resultados podemos concluir que el presente estudio aporta indicios cuantitativos que señalan al Programa de tratamiento como responsable de la mayor parte del cambio medido en los participantes, en línea con lo sostenido por Nezu y Nezu (2005), lo informado por Strunk, Brotman, DeRubeis, y Hollon (2010) o Wiborg, Knoop, Wensing, y Bleijenberg (2013) y, en menor medida, con los resultados de Andersson, Carlbring, y Furmark (2012), pero no de todo el cambio; el efecto del nivel o grado de competencia en el ejercicio de la profesión, cuando se garantiza el conocimiento del tratamiento y la fidelidad o adherencia a su proceso de aplicación, también se muestra relevante aunque sea en menor medida.

Los estudios futuros nos deberían concretar la relevancia de las relaciones puestas de manifiesto en el presente trabajo, hecho por el cual nuestros resultados deben ser contemplados con la cautela que requieren las limitaciones de la presente investigación que afectan, además de a la potencia estadística por el tamaño de nuestra muestra, a la validez externa e interna de nuestra intervención y a la operacionalización realizada sobre la competencia del terapeuta. Así, (i) la validez externa se ve limitada por las características de nuestra muestra, especialmente el rango de edad, su condición de estudiantes y su pertenencia geográfica; (ii) la validez interna se ve amenazada porque no se han controlado las respuestas de escape de orden cognitivo que se hayan podido producir; y (iii) la operacionalización de la competencia del terapeuta también se ve limitada tanto en lo que respecta al gradiente utilizado (un año de diferencia entre las categorías cuasiexperto y experto) como en relación con los requisitos exigidos (experiencia profesional en el tratamiento individual y grupal).

Además, la presente investigación deja pendiente el estudio de la inclusión de la flexibilidad en la aplicación del Programa (Connolly, Crits-Christoph, Levinson y Barber, 2003), el del efecto de la ausencia de control respecto del conocimiento del Programa y la adherencia durante su aplicación (Dobson y Kazantzis, 2003; Kim, Wampold y Bolt, 2006), el de la inclusión de paraprofesionales o el entrenamiento en el manejo de situaciones potencialmente problemáticas previo a su aplicación controlada (Anderson, Ogles, Patterson, Lambert, y Vermeersch, 2009).

Andersson, G., Carlbring, P., y Furmark, T. (2012) Therapist Experience and Knowledge Acquisition in Internet-Delivered CBT for Social Anxiety Disorder: A Randomized Controlled Trial. PLoS ONE 7(5): e37411. Doi:10.1371/journal.pone.0037411

Atienza, F. L., Moreno, Y., y Balaguer, I. (2000). Análisis de la dimensionalidad de la Escala de Autoestima de Rosenberg en una muestra de adolescentes valencianos. Revista de Psicología Universitas Tarraconensis, 22, 29 42 . 
Bados, A. (2009). Fobia Social. Recuperado de http://diposit.ub.edu/dspace/bitstream/2445/6321/1/Fobia\%20socia l.pdf

Beidel, D. C., Turner, S. M. (2005). Childhood Anxiety Disorders. A guide to Research and Treatment. Nueva York: Routledge.

Cella, M., Stahl, D., Reme, S. E. y Chalder, T. (2011). Therapist effects in routine psychotherapy practice: An account from chronic fatigue syndrome. Psychotherapy Research, 21, 168-178.

Connolly Gibbons, M. B., Crits-Christoph, P., Levinson, J., y Barber, J. (2003). Flexibility in manual-based psychotherapies: Predictors of therapist interventions in interpersonal and cognitive-behavioural therapy. Psychotherapy Research, 13, 169-185.

Crits-Christoph, P. y Gallop, R. (2006). Therapist effects in the TDCRP and other psychotherapy studies. Psychotherapy Research, 16, 178-181.

Crits-Christoph, P. y Mintz, J. (1991). Implications of therapist effects for the design and analysis of comparative studies of psychotherapies. Journal of Consulting and Clinical Psychology, 59, 20-26.

Dobson, K. S. y Kazantzis, N. (2003). Role of the therapist in cognitivebehavioural therapy Psychotherapy Research, 13, 131-121.

Echeburúa, E. y Corral, P. (1987). Escala de Inadaptación. Manuscrito no publicado.

Echeburúa, E., Corral, P. y Fernández-Montalvo, J. (2000). Escala de inadaptación (EI): Propiedades psicométricas en contextos clínicos. Análisis y Modificación de Conducta, 26, 325-340.

Elkin, I., Falconnier, L., Martinovitch, Z., y Mahoney, C. (2006). Therapist effects in the NIMH Treatment of Depression Collaborative Research Program. Psychotherapy Research, 16, 144-160.

Gavino, A. (2010). Habilidades del terapeuta. En J. P. Espada, J. Olivares y F. X. Méndez (eds.), Terapia Psicológica. Casos prácticos (pp. 23-44). Madrid: Pirámide.

Gil-Bernal, F. (2009). Evaluación de un modelo terapéutico para el manejo de la fobia social en niños en edad escolar. Tesis doctoral no publicada. Escuela de Psicología. Universidad Nacional Autónoma de México, México D. F.

Gil-Bernal, F y Hernández-Guzmán, L. (2009). Tratamiento cognitivoconductual para niños mexicanos con fobia social. Anuario de Psicología, 40, 89-104.

Gilkinson, H. (1942). Social fears as reported by students in college speech classes. Speech Monographs, 9, 141-160.

Hernández, J. L. (2006). Intervención en adolescentes con fobia social frente a la transmisión de información y grupo de control pasivo. Tesis doctoral no publicada. Facultad de Psicología. Universidad de Murcia.

Hidalgo, M. D., López-Pina, J. A., Inglés, C. J. y Méndez, F. X. (2002). Análisis psicométrico del Cuestionario de confianza para hablar en público, usando la teoría de respuesta al ítem. Anales de psicología, 18, 333-349.

Hofmann, S. G. y DiBartolo, P. M. (2000). An instrument to assess selfstatements during public speaking: Scale development and preliminary psychometric properties. Behavior Therapy, 31, 499-515.

Méndez, F. X., Inglés, C. J., e Hidalgo, M. D. (1999). Propiedades psicométricas del cuestionario de confianza para hablar en público: estudio en una muestra de alumnos de enseñanzas medias. Psicothema, 11, 65-74

Kazantzis, N. (2003). Therapist Competence in Cognitive-behavioural Therapies: Review of the Contemporary Empiral Evidence. Behavior Change, 20, $1-12$.

Kessler, R. C., Berglund, P., Demier, O., Jin, R., Merikangas, K. R. y Walters, E. E. (2005). Lifetime prevalence and age-of-onset distributions of DSM-IV disorders in the National Comorbidity Survey Replication. Archives of General Psychiatry, 62, 593-602.

Kim, D. M., Wampold, B. E. y Bolt, D. (2006). Therapist effects in psychotherapy: A random-effects modeling of the National Institute of Mental Health Treatment of Depression Collaborative Research Program data. Psychotherapy Research, 16, 161-172.

La Greca, A. M. y López, N. (1998). Social anxiety among adolescents: Linkages with peer relations and friendships. Journal of Abnormal Child Psychology, 26, 83-94.

Nezu, A. M., y Nezu, C. M. (2005). Comments on "Evidence-Based Behavioral Medicine: What Is It and How Do We Achieve It?": The Interventionist Does Not Always Equal the Intervention-The Role of Therapist Competence. Annals of Behacioral Medicine, 29 (1), 80.
Ogles, B. M., Lunnen, K. M. y Bonesteel, K. (2001). Clinical significance: History, application, and current practice. Clinical Psychology Review, 21, 421-446.

Okiishi, J. C., Lambert, M. J., Eggett, D. Nielsen, L., Dayton, D. D. y Vermeersch, D. A. (2006). An Analysis of Therapist Treatment Effects: Toward Providing Feedback to Individual Therapists on Their Clients' Psychotherapy Outcome. Journal of Clinical Psychology, 62(9), 1157-117.

Olivares, J. (2009). Evaluación y tratamiento de la fobia social en población infanto-juvenil de los países de habla española y portuguesa. Anuario de Psicología, 40, 7-23

Olivares, J. (dir. y coord.) (2005). Programa LAFS. Protocolo para el tratamiento de la Fobia Social en adolescentes. Madrid: Pirámide.

Olivares, J. García-López, L. J., Hidalgo, M. D., Turner, S. M. y Beidel, D. (1999). The Social Phobia and Anxiety Inventory: Reliability and validity in an adolescent Spanish population. Journal of Psychopathology and Behavioral Assessment, 21 (1), 67-78.

Olivares, J., García-López, L. J., Turner, S., Beidel, D., Albano, A. M. e Hidalgo, M.D. (2002). Results at long-term among three psychological treatments for adolescents with social phobia (I): Statistical significance. Psicologia Conductual, 10, 147-164.

Olivares, J., García-López, L. J., Turner, S., La Greca, A. M. y Beidel, D. (2002). A pilot study on normative data for two social anxiety measures: The Social Phobia and Anxiety Inventory and the Social Anxiety Scale for Adolescents. International Journal of Clinical and Health Psychology, 2, 467-476.

Olivares, J., Rosa-Alcázar, A. I., Caballo, V. E., García-López, L. J., Orgiles, M. y López-Gollonet, C. (2003). Tratamiento de la fobia social en niños y adolescentes: una revisión meta-analítica. Psicología Conductual, 11 (3), 599-622.

Olivares, J., Rosa-Alcázar, A. I. y Olivares-Olivares, P. J. (2006). Relevancia de la atención individualizada en el tratamiento en grupo de adolescentes con fobia social generalizada. International Journal of Clinical and Health Psychology, 6, 565-580.

Olivares-Olivares, P. J., Rosa-Alcázar, A. I. y Olivares, J. (2008). Does individual attention improve de effect of group treatment of adolescents with social phobia? International Journal of Clinical and Health Psychology, 8, 465-481.

Olivares-Olivares, P. J., Rosa-Alcázar, A. I. y Olivares, J. (2007). Validez social de la intervención en adolescentes con fobia social: padres frente a profesores. Terapia Psicológica, 25, 63-71.

Olivares, J., Ruiz, J., Hidalgo, M. D., García-López, L. J., Rosa-Alcázar, A. I. y Piqueras, J. A. (2005). Social Anxiety Scale for Adolescents (SAS-A): psychometric properties in a Spanish-speaking population. International Journal of Clinical and Health Psychology, 5, 85-97.

Rivero, R. (2005).Validación de tres instrumentos para la medida de la fobia social en la adolescencia. Tesis doctoral no publicada. Facultad de Psicología. Universidad de Salamanca.

Rosenberg, M. (1965). Society and the Adolescent Self Image. Princeton: Princeton University Press.

Silverman, W. y Albano, A. M. (1996). Anxiety Disorders Interview Schedule for DSM-IV. Child Interview Schedule. (ADIS-IV-C). Nueva York: Graywind Publications.

Silverman, W., Saavedra, L., y Pina, A. (2001). Test-retest reliability of anxiety and diagnoseswith the Anxiety Disorders Interview Schedule for DSM-IV: Child and parent version. Journal of the American Academy of Child and Adolescent Psychiatry, 40, 937-944.

Silverman, W.K., Albano, A.M., y Sandín, B. (2001). Entrevista clínica ADISIV-C: entrevista para el niño [ADIS-IV-C Clinical Interview. Interview Schedule for Children]. Madrid: UNED-Klinik.

SSPS 14 (2007). Statistical Package for the Social Sciences. SPSS Inc.

Strunk, D. R., Brotman, M. A., DeRubeis, R. J., y Hollon, S. D. (2010). Therapist competence in cognitive therapy for depression: Predicting subsequent symptom change. Journal of consulting and clinical psychology, $78(3), 429$

Turner, S. M., Beidel, D. C., Dancu, C. V. y Stanley, M. A. (1989). An empirically derived inventory to measure social fears and anxiety: The Social Phobia and Anxiety Inventory. Psychological Assessment, 1, 35-40.

Vallés, A. (2005). Intervención en Adolescentes con Fobia Social Generalizada frente a los Grupos de Control Activo y Lista de Espera. Tesis doctoral no publicada. Facultad de Psicología. Universidad de Murcia. 
Wampold, B. E. y Bolt, D. M. (2006). Therapist effects: Cléver ways to make them (and everything else) disappear. Psychotherapy Research, 16 (2), $184-$ 187.

Wiborg, J., Knoop, H., Wensing, M., y Bleijenberg, G. (2012). Therapist effects and the dissemination of cognitive behavior therapy for chronic fatigue syndrome in community-based mental health care. Behaviour Research and Therapy, 50(6), 493-406. Doi: 10.1016/j.brat.2012.03.002.
Wilkins, V. M., Nezu, A. M. y Nezu, C. M. (2001). Treatment integrity in CBT: A 10-year "report card". 35th Annual Meeting of the Association for Advancement of Behavior Therapy. Philadelphia.

(Articulo recibido: 5-12-2012; revisión recibida: 16-3-2013; aceptado: 25-9-2013) 